\title{
Acute Pyelonephritis
}

\begin{abstract}
Acute pyelonephritis is an infection of upper urinary tract and results from bacterial invasion of the kidney. E coli are the most common pathogen involved. A meticulous history and physical examination supplemented with urinalysis can diagnose it early and may be life saving for patients. This brief review is an attempt to discuss the current etiopathogenesis and management of acute pyelonephritis.
\end{abstract}

Keywords: Pyelonephritis; Urinary tract infections
Volume 5 Issue 5 - 2017

\section{Ramesh Aggarwal,' Anshuman Srivastava,' Sachin Kumar Jain,' Meenakshi Aggarwal' \\ 'Department of Medicine, Lady Hardinge Medical College \& Associated Hospitals, India \\ ${ }^{2}$ Specialist Microbiology, Kalawati Saran Children Hospital, India}

Correspondence: Ramesh Aggarwal,Associate Professor, Department of Medicine, Lady Hardinge Medical College \& Associated Hospitals, New Delhi I I000I, India

Email rameshlhmc@gmail.com

Received: September 29, 2017 | Published: November 13, 2017

\section{Introduction}

Urinary tract infections (UTIs) include cystitis and pyelonephritis. Acute pyelonephritis is an infection of the upper urinary tract, specifically the renal parenchyma and renal pelvis. Common organisms causing pyelonephritis are discussed in Table 1. Acute pyelonephritis is considered uncomplicated if the infection is caused by a typical pathogen in an immunocompetent patient who has normal urinary tract anatomy and renal function. A complicated pyelonephritis is said to be present when it occurs in a patient with a structural or functional abnormality of the genitourinary tract and it leads to increase in the risk of unsuccessful treatment. In the United States there are approximately 250,000 cases of acute pyelonephritis each year, resulting in more than 100,000 hospitalizations. ${ }^{1,2}$ Women are approximately five times more likely than men to be hospitalized with this condition however they have less mortality rates than men attributable to pyelonephritis. ${ }^{2}$

Table I Causative Organisms in Acute Pyelonephritis2

\begin{tabular}{|l|l|}
\hline Organism & Prevalence \\
\hline Escherichia coli & $\begin{array}{l}83 \% \text { (Women) } \\
74 \% \text { (Men) }\end{array}$ \\
\hline Klebsiella pneumonia & $2.7 \%$ (Women) \\
& $6.2 \%$ (Men) \\
\hline Staphylococcus saprophyticus & $<3 \%$ (Women) \\
\hline Candida species & \\
\hline Enterococcus species & Rare \\
\hline Other Enterobacteriaceae & Rare \\
(e.g., Proteus species, Enterobacter species) & Rare \\
\hline $\begin{array}{l}\text { Pseudomonas aeruginosa } \\
\text { Ureaplasma species }\end{array}$ & \\
\hline
\end{tabular}

Risk Factors for Complicated Acute Pyelonephritis. ${ }^{1,3}$

i. Age: Infants, Elderly ii. Immunosuppressed state: Diabetes mellitus, Malignancy, HIV infection

iii. Obstruction: Calculi, Foreign body, Anatomic/functional abnormality, Transplantation, Bladder neck obstruction, Posterior urethral valve, Horseshoe kidney, benign prostatic hypertrophy, neurogenic bladder.

iv. Pregnancy

v. Male sex

vi. Instrumentation.

\section{Clinical Features}

The clinical spectrum of acute pyelonephritis is wide, ranging from a mild illness to sepsis syndrome. Mild pyelonephritis can present as low-grade fever with or without lower-back or costovertebral-angle pain, whereas severe pyelonephritis can manifest as high fever, rigors, nausea, vomiting, and flank and/or loin pain. Symptoms are generally acute in onset, and symptoms of cystitis may not be present. Fever is a distinguishing feature between cystitis and pyelonephritis. Symptoms suggestive of cystitis (dysuria, urinary bladder frequency and urgency, and suprapubic pain) may also be present. ${ }^{1}$ In diabetics a rare but dreadful complication might occur which is called as emphysematous pyelonephritis. This is an acute and severe infection of the renal parenchyma and peri-renal tissue, which results in gas within the renal parenchyma, collecting system or perinephric tissue. It is a life threatening condition if not treated promptly. ${ }^{3}$ Xanthogranulomatous pyelonephritis occurs in association with chronic urinary obstruction (usually by staghorn calculi), together with chronic infection. It leads to granulomatous inflammation and destruction of renal tissue. ${ }^{4}$

\section{Diagnostic Evaluation}

Complete blood count and urinalysis is the initial investigations whenever pyelonephritis is suspected. Blood glucose estimation should be done to rule out diabetes mellitus. Serum urea and creatinine should be measured to assess the renal functions, especially in cases of complicated pyelonephritis. In urinalysis either nitrite or leukocyte esterase positivity can be interpreted as a positive result. Urine microscopy reveals pyuria in nearly all cases of cystitis and haematuria in approximately $30 \%$ of cases. Blood in the urine also may suggest a possibility of UTI. The growth of bacteria in a urine 
culture is the diagnostic "gold standard" for UTI.A quantitative count of at least $10^{5} \mathrm{cfu} / \mathrm{dL}$ in a voided specimen is consistent with infection in the non catheterized patient, whereas a count of $>10^{3} \mathrm{cfu} /$ $\mathrm{dL}$ is sufficient for the diagnosis in case of patients with indwelling urinary catheters. ${ }^{5}$ Ultrasound abdomen should be done whenever a complicated pyelonephritis is suspected. ${ }^{1,6}$

\section{Management}

Outpatient oral therapy is successful in 90 percent of selected patients with uncomplicated acute pyelonephritis who can tolerate oral intake, will be compliant with the treatment regimen, will return for early follow-up, and have adequate social support Patients with complicated acute pyelonephritis who are more ill or have not responded to outpatient therapy should be hospitalized.'

\section{Indications for Hospitalization in Patients with Acute Pyelonephritis $^{7}$}

I. Absolute indications: Persistent vomiting, progression of uncomplicated UTI, suspected sepsis, urinary tract obstruction.

II. Relative indications: Age $>60$ years, anatomic urinary tract abnormality, immunocompromised status (diabetes mellitus, HIV, neoplasia, organ transplant).

Since pyelonephritis is a tissue-invasive disease, the treatment regimen chosen should aim at eradicating the causative organism and should reach optimum therapeutic blood levels quickly. Fluoroquinolones are the first-line therapy for acute uncomplicated pyelonephritis due to $E$ Coli. Fluoroquinolones can be given orally or parenterally and it depends on the patient's tolerance for oral intake. A randomized clinical trial demonstrated that a 7-day course of therapy with oral ciprofloxacin $(500 \mathrm{mg}$ twice daily, with or without an initial IV 400-mg dose) was highly effective for the initial management of pyelonephritis in the outpatient setting. ${ }^{8}$ Oral TMP-SMX (one doublestrength tablet twice daily for 14 days) also is effective for treatment of acute uncomplicated pyelonephritis if the uropathogen is known to be susceptible. Nitrofurantoin $100 \mathrm{mg} \mathrm{q} 12 \mathrm{~h}$ can also be used in cases of susceptible organisms. ${ }^{9}$

If the pathogens susceptibility is not known and trimethoprimsulphamethoxazole is used, an initial IV 1-g dose of ceftriaxone is recommended. Oral $\beta$-lactam agents are less effective than the fluoroquinolones and should be used with caution and close followup. Options for parenteral therapy for uncomplicated pyelonephritis include fluoroquinolones, an extended-spectrum cephalosporin with or without an aminoglycoside, or a carbapenem. Aminoglycosides should be avoided in patients with pre-existing renal disease. In patients with more complicated histories, previous episodes of pyelonephritis, or recent urinary tract manipulations, combinations of a $\beta$-lactam and a $\beta$-lactamase inhibitor (e.g., ampicillin-sulbactam, ticarcillinclavulanate, piperacillin-tazobactam) or imipenem-cilastatin can be used; as a rule the treatment of such patients should be based on urine culture results. Amoxicillin or amoxicillin-clavulanate potassium is preferred during pregnancy and in the treatment of infections caused by gram-positive organisms. A seven- to 14-day course of antibiotics is effective in women who are immunocompetent and do not have underlying illness, complicated UTI may warrant longer course of treatment. ${ }^{10}$

Fever generally resolves within 72 hours of starting antibiotic therapy. Oral treatment is feasible as soon as the patient becomes a febrile, has improved clinically, and can tolerate oral hydration and medications. It is not necessary to use the same agent for both parenteral and oral therapy. Post-treatment urine cultures are recommended in all patients at the follow-up visit, one to two weeks after completion of antibiotic therapy. Complication like emphysematous pyelonephritis is usually treated with percutaneous drain or nephrectomy. ${ }^{11}$ If recurrence of infection or relapse (reinfection less than 14 days after completing an appropriate regimen) occurs, the patient needs to undergo systematic evaluation for anatomic, functional, or structural abnormalities.

\section{Conclusion}

Acute Pyelonephritis is a tissue invasive disease and should be treated promptly so as to prevent renal damage. Life threatening complications like emphysematous pyelonephritis should be anticipated in diabetic patients and managed without delay.

\section{Acknowledgments}

None.

\section{Conflicts of Interset}

None.

\section{References}

1. Ramakrishnan K, Scheid DC (2005) Diagnosis and management of acute pyelonephritis in adults. Am Fam Physician 71(5): 933-942.

2. Czaja CA, Scholes D, Hooton TM, Stamm WE (2007) Population-based epidemiologic analysis of acute pyelonephritis. Clin Infect Dis 45(3): 273-280.

3. Shields J, Maxwell AP (2010) Acute pyelonephritis can have serious complications. Practitioner 254(1728): 19, 21, 23-24.

4. Li L, Parwani AV (2011) Xanthogranulomatous pyelonephritis. Arch Pathol Lab Med 135(5): 671-674.

5. Rubin RH, Shapiro ED, Andriole VT, Davis RJ, Stamm WE (2003) Evaluation of new anti-infective drugs for the treatment of urinary tract infection. Infectious Diseases Society of America and the Food and Drug Administration. Clin Infect Dis 15: 216-227.

6. Enikeev DV, Glybochko P, Alyaev Y, Enikeev M, Rapoport L (2017) Imaging technologies in the diagnosis and treatment of acute pyelonephritis. Urologia 84(3): 179-184.

7. Bass PF, Jarvis JA, Mitchell CK (2003) Urinary tract infections. Prim Care 30(1): 41-61.

8. McCarty JM, Richard G, Huck W, Tucker RM, Tosiello RL, et al (1999) A randomized trial of short-course ciprofloxacin, ofloxacin, or trimethoprim/sulfamethoxazole for the treatment of acute urinary tract infection in women. Ciprofloxacin Urinary Tract Infection Group. Am J Med 106(3): 292-299.

9. Tasbakan MI, Pullukcu H, Sipahi OR, Yamazhan T, Ulusoy S (2012) Nitrofurantoin in the treatment of extended-spectrum $\beta$-lactamaseproducing Escherichia coli-related lower urinary tract infection. Int $\mathrm{J}$ Antimicrob Agents 40(6): 554-556.

10. Roberts JA (1999) Management of pyelonephritis and upper urinary tract infections. Urol Clin North Am 26(4): 753-763.

11. Sokhal AK, Kumar M, Purkait B, Jhanwar A, Singh K, et al. (2017) Emphysematous pyelonephritis: Changing trend of clinical spectrum, pathogenesis, management and outcome.Turk J Urol 43(2): 202-209. 